\title{
The North Shore-Long Island Jewish Research Institute
}

The North Shore-Long Island Jewish Research Institute is directed by Nicholas Chiorazzi, MD. It provides investigators from a variety of scientific fields and backgrounds with the opportunity to focus on disease-oriented medical research. The goal of the institute is to understand the underlying biological processes of disease and ultimately to develop new and more effective therapies for patients. The affiliation with the North Shore-Long Island Jewish Health System and its patients provides a unique opportunity for focused biomedical and translational research.

\section{HISTORY}

In 1999, the North Shore-Long Island Jewish Health System, in recognition of the critical importance of this research to the larger health system mission, created the Research Institute as a separate entity. The goal was to better foster the growth and development of ongoing efforts in the biomedical sciences. The Institute has over 125 members actively working on biomedical research. The Research Institute provides a fertile environment for the expansion of research programs, as well as core scientific support. The overall research program receives $\$ 26,000,000$ in federal funding.

\section{CENTERS OF EXCELLENCE}

Institute scientists currently focus their efforts on four scientific areas-genomics and human genetics, neurosciences, immunology and inflammation, and oncology and cell biology.

\section{The Robert S Boas Center for Genomics \& Human Genetics}

The completion of the Human Genome Project vastly expands the number and types of opportunities in biomedical research through the next decade and beyond. Major impact on medical progress in the future will result from understanding the genetic bases of both more common and genetically complex illnesses such as cancer, autoimmunity, heart disease, and mental disorders. Investigators in the Boas Center for Genomics \& Human Genetics, directed by P Gregersen, MD, recently have developed a series of important insights regarding the genetic susceptibility of individuals to rheumatoid arthritis and other autoimmune diseases. The center is bolstered by a large clinical trials program at North Shore University Hospital and Long Island Jewish Medical Center, which serve as a natural resource for these studies. Currently, large-scale genetic mapping projects, including studies related to autoimmunity, twinning, and neurocognitive traits and disorders are underway.

The Susan \& Leonard Feinstein Center for Neuroscience The Susan \& Leonard Feinstein Center for Neuroscience, directed by $\mathrm{D}$ Eidelberg, MD, is dedicated to the study of neurodegenerative and neuropsychiatric illnesses. A major focus of the center is the study of mechanisms that underlie neural recuperation fol- lowing brain injury associated with neurodegeneration, genetic disorders, stroke, and trauma. Major contributions already have been made in deciphering the neural pathways involved in Parkinson's disease. Research within the center is generating information important for the development of new pharmacological and surgical approaches to treat these conditions through the integration of state-of-the-art techniques of brain imaging, neurophysiology, neurochemistry, and cognitive neuroscience.

\section{The Center for Immunology \& Inflammation}

The Center for Immunology \& Inflammation, directed by S Pahwa, MD, and N Chiorazzi, MD, focuses on understanding the basic biologic principles that underlie the immune and inflammatory systems in health and disease. The major conditions under study include immune deficiency disorders (for example, congenital and secondary immune deficiencies and AIDS), autoimmune disorders (for example, rheumatoid arthritis and systemic lupus erythematosus), inflammatory bowel disease (for example, Crohn's disease and ulcerative colitis), and chronic and acute systemic inflammatory disorders, many of which involve abnormalities of the cytokine and chemokine network (for example, septic shock and adult respiratory distress syndrome).

\section{The Center for Oncology \& Cell Biology}

The Center for Oncology \& Cell Biology, directed by I Goldberg, $\mathrm{MD}$, and B Steinberg, PhD, is dedicated to providing interactive and collaborative research for the understanding of the molecular mechanisms of cell and tissue function in health and disease. Projects include the study of genetic mutations and epigenetic modifications associated with the development of breast and prostate cancer, papillomavirus-initiated premalignant tumors, angiogenesis, cell signaling in carcinogenesis, chemoprevention and the identification of new biological markers as prognostic indicators in malignancy, such as chronic lymphocytic leukemia. Additional programs focus on molecular cardiovascular function and disorders, urogenital function and disorders, and the role of nutrition and micronutrients in disease.

\section{The Center for Patient-Oriented Research}

The mission of the Center for Patient-Oriented Research, directed by $\mathrm{K}$ Tracey, MD, is to bring the fruits of scientific research to the bedside by providing a resource center to facilitate the discovery 
and development of new therapeutics and diagnostics for human disease. The Center integrates expertise from broadly defined areas, including drug discovery and bioassays, medicinal chemistry, pharmacology and pharmacokinetics, physiology, toxicology, regulatory, and clinical trials design. Patients participate directly in research studies though the activities of the General Clinical Research Center (GCRC) within the Center for PatientOriented Research. The GCRC facilitates the process of clinical research by providing a research facility with examination rooms, research nurses, core laboratory facilities (imaging, molecular genetics, biostatistics, and sample acquisition), and training in clinical research.

\section{A NEW MISSION AND COMMITMENT}

As the research institute continues to expand its scientific and clinical missions, we believe that these efforts complement our commitment to provide the means by which important advances in the biomedical sciences are disseminated to the scientific community.
Molecular Medicine provides just such a forum and we are pleased to have the opportunity to support and promote its activities.

\section{SELECTED PUBLICATIONS}

1. Gregersen PK. (1999) Generics of rheumatoid arthritis: confronting complexity. Arthritis Research. 1:37-44.

2. Damle RN et al. (1999) Ig $\vee$ gene mutation status and CD38 expression as novel prognosis indicators in chronic lymphocytic leukemia. Blood. 94:1840-7.

3. Wang $\mathrm{H}$ et al. (1999) HMG-1 as a late mediator of endotoxin lethality in mice. Science. 285:248-51.

4. Fan $S$ et al. (1999) BRCAI inhibits estrogen receptor signaling in transfected cells. Science. $284: 1354-6$

5. Jin L et al. (1999) Indole-3-carbinol prevents cervical cancer in HPV16 transgenic mice. Cancer Res. 59:3991-7.

6. Tateyana M, Oyaizu N, McCloskey TW, Than S, Pahwa S. (2000) CD4 T Iymphocytes are primed to express Fas ligand by CD4 cross-linking and contribute to CD8 T cell apoptosis via Fas/FasL death signaling pathway. Blood. 96:195-202

7. Tracey KJ. (2002) The inflammatory reflex. Nature. Review. 420:853-9.

8. Gurrieri C et al. (2002) Chronic lymphocytic leukemia B cells can undergo somatic hypermutation and intraclonal immunoglobulin $\mathrm{V}(\mathrm{H}) \mathrm{DJ}(\mathrm{H})$ gene diversification. J. Exp. Med. 196(5):629-39.

9. Ivanova S et al. (2002) Neuroprotection in cerebral ischemia by neutralization of 3-aminopropanal. Proc. Natl. Acad. Sci. U.S.A. 99(8):5579-84

10. Wang $\mathrm{H}$ et al. (2003) Nicotinic acetylcholine receptor a7 subunit is an essential regulator of inflammation. Nature. 421:384-8. 